\title{
Time-of-flight MeV-SIMS with beam induced secondary electron trigger
}

\author{
Martina Schulte-Borchers, Max Döbeli, Arnold Milenko Müller, Matthias George, Hans-Arno \\ Synal
}

Ion Beam Physics, ETH Zurich, Otto-Stern-Weg 5, CH-8093 Zürich, Switzerland

\begin{abstract}
A new Time-of-flight MeV Secondary Ion Mass Spectrometry (MeV-SIMS) setup was developed to be used with a capillary microprobe for molecular imaging with heavy primary ions at MeV energies. Due to the low output current of the ion collimating capillary a time-of-flight (ToF) measurement method with high duty cycle is necessary. Secondary electrons from the sample surface and transmitted ions were studied as start signals. They enable measurements with a continuous primary beam and unpulsed ToF spectrometer. Tests with various primary ion beams and sample types have shown that a secondary electron signal is obtained from 30 to $40 \%$ of incident $\mathrm{MeV}$ particles. This provides a ToF start signal with considerably better time resolution than the one obtained from transmitted primary ions detected in a radiation hard gas ionization detector. Beam induced secondary electrons therefore allow for MeVSIMS measurements with reasonable mass resolution at primary ion beam currents in the low fA range.
\end{abstract}

\section{Introduction}

Molecular imaging by mass spectrometric techniques is an indispensable tool in biomedical research (e.g. [1, 2]). Secondary ion yields for macromolecules and large molecular fragments are strongly enhanced if fast and heavy primary ion beams are used in Secondary lon Mass Spectrometry (SIMS) [3, $4,5]$. Nearly intact molecules have been reported to leave the surface under these conditions, which considerably facilitates identification of chemical species. In past years, the use of MeV ions for ToFSIMS has been investigated for molecular imaging with very promising results and the technique has been applied to imaging of biological samples $[6,7]$.

One major difficulty in MeV-SIMS for imaging is the limited focusing ability of typical magnetic quadrupole lenses for heavy and fast primary beams. To a certain extent electrostatic lenses can be applied at the expense of lower focusing quality. As an alternative to ion optical lens systems, a MeVSIMS setup based on a capillary microprobe [8] was developed at ETH Zurich. Such a capillary acts as a collimator for a wide variety of primary beams including very heavy and fast ions, which are the most promising candidates to exploit the full potential of accelerator based MeV-SIMS. Beam spot diameters down to about 1 micron are possible, but as a result of the strong collimation the achieved beam current is relatively low. However, currents in the fA to pA range, usually obtained with capillaries, are sufficient for MeV-SIMS due to the high secondary ion yields.

For ToF mass spectrometry, on the other hand, a precise start signal as an estimate for impact time of the primary ion on the sample or a pulsed secondary mass spectrometer is necessary. Several ToF MeV- 
SIMS systems use a fast primary beam chopper to produce a start signal, e.g. [5, 9, 10]. The time resolution is then defined by the duration of the primary particle pulse, which can be as short as a few nanoseconds for modern devices [9]. A big drawback of all pulsed systems is their small duty cycle since the flight times of heavy secondary ions are several tens of microseconds and the waiting period between beam bunches has to be larger than this. For highly focused beams with low brightness and especially for low intensity primary species such as molecular or cluster ions, the DC currents generated in tandem accelerators are already reduced to the $f A$ level due to the charge stripping process [11]. Additional beam pulsing results in count rates that are often too low for reasonable imaging applications. For a capillary microbeam the same problem applies, since the primary beam is additionally reduced by several orders of magnitude by collimation directly in front of the sample. Bunched spectrometers [12] are a valid alternative to primary beam pulsing as they can be used with DC primary beams and analyze secondary ions with dramatically improved duty cycle. They are, however, an important cost factor in an MeV-SIMS system and require careful adaptation in a customized environment due to their advanced sophistication. Therefore an alternative high duty cycle ToF trigger technique is desirable for imaging with a capillary microprobe. In early systems working with fission sources the recoil nucleus was used to produce the ToF start signal [3] which is no option in accelerator based setups. For thin samples the transmitted primary ion can be detected [13]. However, this approach complicates sample preparation and limits the applicability of the technique. In addition, the primary ion detector can suffer from radiation damage very fast. In the case of negative secondary ion detection, the secondary electrons emitted by the sample surface can be used to produce a fast start signal in the stop detector of the ToF spectrometer [6,9]. Alternatively also photons produced by primary ion impact have been shown to present fast start signals for high resolution ToF measurements [14], but the design of an experiment with reliably high light detection efficiency is rather difficult and the background in spectra taken with a photon trigger is therefore increased.

In the present work we introduce the method of secondary electrons from the sample to be used as ToF start signal in positive ion MeV-SIMS. The setup was designed to allow extracting secondary electrons on one side of the sample, while positive secondary ions are extracted to the opposite side. The results of this new technique are compared with beam pulsing and transmitted ions as start signals in the same ToF MeV-SIMS system at the Laboratory of Ion Beam Physics at ETH Zurich. Performance of the setup is evaluated in terms of efficiency and time resolution with different start signals. The full details of the new MeV-SIMS set-up including imaging capabilities will be presented in a later publication.

\section{Experimental}

All experiments were conducted at ETH Zurich in the Laboratory of Ion Beam Physics, whose $6 \mathrm{MV}$ tandem accelerator facility is used for Accelerator Mass Spectrometry (AMS) and materials analysis and modification $[15,16]$. The MeV-SIMS set-up is attached to a part of the AMS beam line which includes only electrostatic ion optical elements on the high energy side of the accelerator. This allows to use ion beams of high mass at low charge states, such as accelerated $\mathrm{C}_{60}$ clusters of up to $18 \mathrm{MeV}$ [11] and monoatomic heavy ions like iodine or gold of up to nearly $80 \mathrm{MeV}$. In addition, beam pulsing is available from AMS applications [17]. For the higher timing requirements of MeV-SIMS, pulse length was adjusted to be as short as possible. Its performance will be evaluated later. 
Dedicated for MeV-SIMS, a new beamline and UHV chamber (see Fig. 1) was added to the $0^{\circ}$ port of the high energy AMS magnet. Without baking, the chamber reaches a base pressure around $1 \cdot 10^{-7} \mathrm{mbar}$ and includes a load lock for a tray with five samples. The setup is designed for imaging with a capillary microbeam and a piezo raster stage [8]. In order to simplify the study of different ToF trigger modes the capillary was not mounted for the measurements presented here.

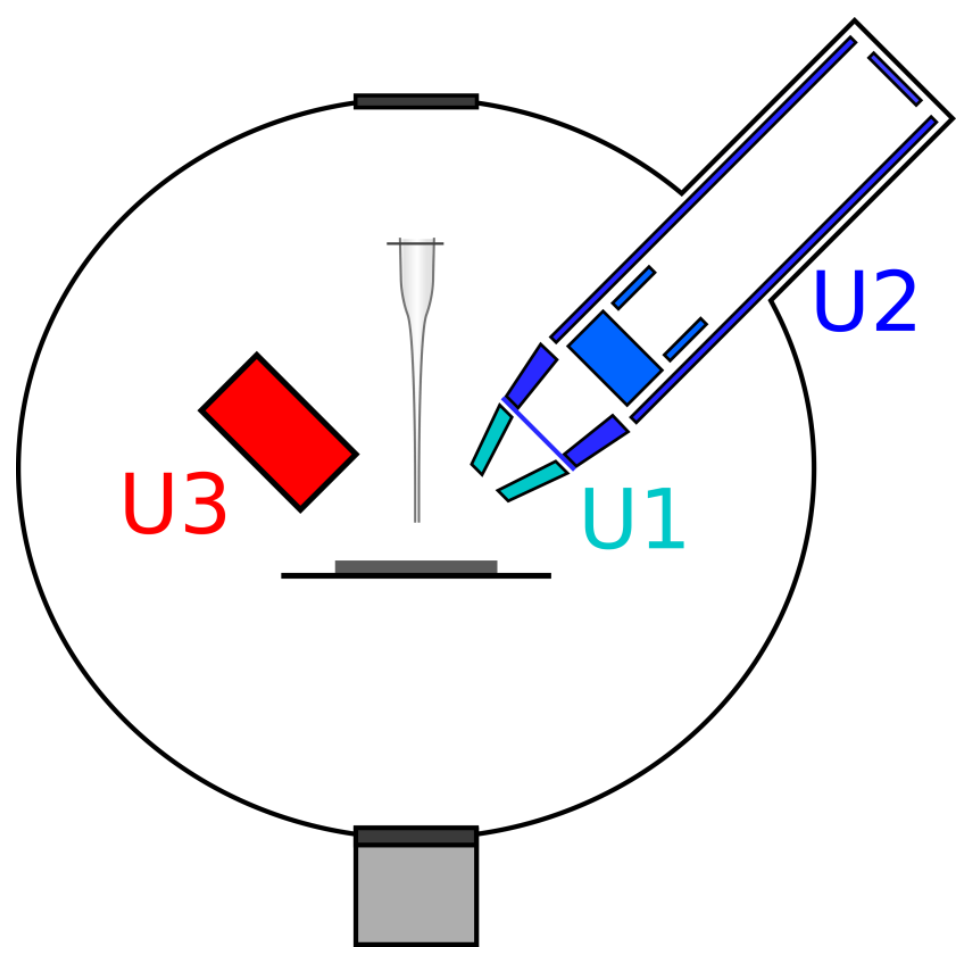

Fig. 1: Top view of the new MeV-SIMS chamber, with the primary beam coming from the top. The sample is placed on the piezo table in the middle. In front of the sample are an electron detector (left) with positive voltage $U_{3}$ and a Time-of-flight tube (right) with voltages $U_{1}$ and $U_{2}$. Behind the sample is a gas ionization detector.

For optimization of simultaneous positive secondary ion and secondary electron extraction the inner setup of the chamber was simulated in detail using SIMION ion trajectory software [18] version 8.0.3. Primary ions hit the sample along the surface normal, while the extracting nozzle of the ToF tube and the electron detector are on either side $45^{\circ}$ from the primary beam in the horizontal plane. For electron extraction and detection a channeltron (SJUTS KBL505) is used with positive bias voltage of a few kV. The ToF spectrometer has a two stage extraction system with adjustable negative voltages of -1 to $-2 \mathrm{kV}$ at the nozzle and -5 to $-6 \mathrm{kV}$ at the second stage. Behind the two extraction stages a set of electrostatic $\mathrm{XY}$ steerers is placed for correction of the ion flight path direction. This is necessary due to the angled extraction. The whole flight tube has an isolated metal liner which can be held at a separate potential. For the experiments described here the liner was electrically connected to the second extraction stage. A chevron pair of micro channel plates (Hamamatsu F4293-07) with $20 \mathrm{~mm}$ active diameter serves as stop detector at the end of the $455 \mathrm{~mm}$ long flight tube. The positive voltage at the electron detector and the negative voltage at the ToF nozzle have to be carefully adjusted. Since the electric field direction is perpendicular to the surface for conducting samples the field strength at the point of impact of the 
primary ion beam on the sample has to be low to allow the simultaneous extraction of positively and negatively charged particles.

Furthermore a radiation hard gas ionization detector [19] is mounted in transmission geometry behind the sample for precise measurement of the incoming particle rate in the case of thin samples. Thick samples have to be periodically removed to measure the primary ion rate. The transmission detector can be used as an alternative start signal for thin samples.

The signals of all three detectors as well as the timing signal of the beam pulsing system are connected to a four channel CAEN DT5751 digitizer [20]. Due to the acquisition rate of $1 \mathrm{GS} / \mathrm{s}$ per channel, the intrinsic timing resolution without interpolation is $1 \mathrm{~ns}$ for acquired spectra. This fully digital approach allows the recording of timestamps (and optionally pulse heights) for all four signals in parallel via a custom made LabVIEW application in a straightforward manner. This application controls data acquisition and data analysis simultaneously, including the evaluation of multi-stop events.

\section{Measurement Modes}

The system was designed to enable multiple modes for MeV-SIMS measurements, depending on the sample. This makes a thorough evaluation of the performance possible.

a. Pulsing mode: Primary beam is pulsed and the pulsing signal is used as start for the Time-offlight measurement.

b. Electron start mode: Start time is measured by secondary electrons from the sample, which are detected nanoseconds after the primary ion hit.

c. Transmission mode: For thin samples, primary ions can be detected in the transmission detector behind the sample.

In all cases, positive secondary ions are extracted into the nozzle of the Time-of-flight tube and detected in the MCP detector, independently of the start measurement mode. For thin samples the measurement can even be conducted in all three start modes simultaneously.

For electron start and transmission mode the primary beam current from the accelerator had to be strongly reduced, as no capillary collimator was present. Typical beam currents needed are a few fA i.e. on the order of $10^{4}$ ions per second.

In pulsing mode the beam is electrostatically chopped at the low energy injection magnet. The start time is thereby defined via a signal from the pulsing system, which is recorded by the digitizer. The uncertainty of the actual primary ion impact time is given by the beam pulse length, which determines time resolution. In transmission mode a significant start time jitter is expected from signal generation in the gas detector. For the presented electron start mode the uncertainty of the start time measurement is mainly caused by the variance of the extraction delay in the low electric field area near the sample and the fluctuation of electron travel time to the channeltron.

\section{Results and discussion}


First spectra obtained in electron start mode are presented and their time resolution and efficiency evaluated. Then the performance is compared to Pulsing and Transmission start mode.

\subsection{Electron start mode}

A positive ion mass spectrum taken with electron start signal of a solid, uncleaned gold sample is shown in Fig. 2. A large number of peaks are due to contaminating organics on the surface of the metal and are hard to be readily identified. Assuming that the three lowest mass peaks correspond to $\mathrm{H}^{+}, \mathrm{H}_{2}{ }^{+}$, and $\mathrm{H}_{3}{ }^{+}$ additional peaks can be assigned to $\mathrm{Na}^{+}, \mathrm{K}^{+}$and $\mathrm{Ca}^{+}$to obtain a first mass calibration of the time spectrum.

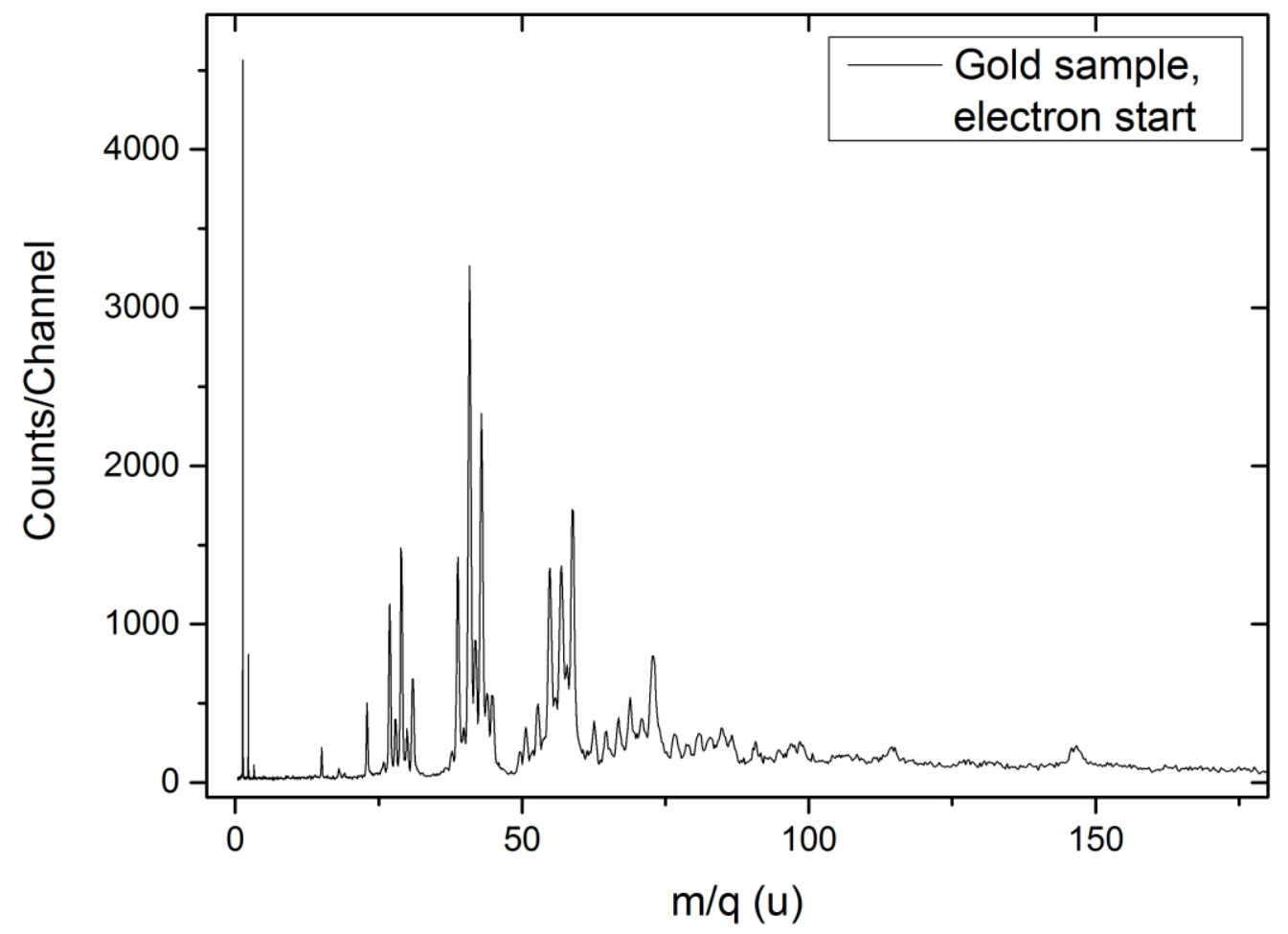

Fig. 2: Positive secondary ion mass spectrum of a solid gold sample produced with 28 MeV Au primaryions, taken in electron start mode.

Time resolution can be evaluated on the $\mathrm{H}^{+}$peak, whose FWHM has been determined to be about $8 \mathrm{~ns}$. With optimum tuning of the spectrometer a mass resolution $\mathrm{m} / \Delta \mathrm{m}$ of 107 has been measured in electron start mode. $\mathrm{m} / \Delta \mathrm{m}$ is approximately constant over the whole investigated mass range, i.e. mass peaks broaden linearly with mass. This points towards the influence of the initial kinetic energy spread of secondary ions. An intrinsic uncertainty of the flight time measurement would rather produce a square root dependence of the mass resolution with mass. The effect of the initial energy distribution of secondary ions on resolution is especially severe due to the low extraction field at the point of emission. 
The time jitter of the electron start signal is most likely much smaller than the experimentally determined 8 ns since it was only measured against the ToF signal of secondary ions. The combined total spread in flight time measurement is probably strongly dominated by the secondary ions. Due to the faster velocity of electrons the influence of the low extraction field on start signal timing is much less severe. SIMION simulations show that for secondary electrons with an initial kinetic energy range of 0 to $5 \mathrm{eV}$ the width of the arrival time distribution of electrons at the channeltron detector is significantly smaller than $1 \mathrm{~ns}$.

To assess extraction and detection efficiencies of electron detector and Time-of-flight system, rates at the respective detectors were compared to the primary ion rate. For thin samples transmitted ions were simultaneously counted in the gas ionization detector to exactly monitor the incoming beam current and to determine coincidence rates with the secondary particle detectors. For thick samples the beam current was measured with the transmission detector before and after short MeV-SIMS measurements by removing the samples with the stage. Since absolute sputter yields of positive secondary ions are not known under MeV-SIMS conditions, such measurements can only give a rough estimate of the detection efficiency of the ToF spectrometer. Not only the number of secondary ions and electrons emitted per primary ion varies with sample composition but extraction efficiencies can also significantly depend on sample properties like surface roughness and conductivity.

Measurements were done on a $2 \mu \mathrm{m}$ thick aluminum foil, a $50 \mathrm{~nm}$ thick SiN foil, gold and PTFE bulk samples as well as a Si wafer sample covered with vacuum-evaporated Leucine. For thin samples, 30 to $40 \%$ of primary ion hits have a valid secondary electron start signal. For 7 to $12 \%$ of all transmission hits at least one secondary ion was detected in the ToF detector within a coincidence time of $20 \mu \mathrm{s}$. For bulk samples, 5 to $45 \%$ of all detected secondary ions have a valid secondary electron start signal, strongly depending on the target material. The highest values were obtained with Leucine which is in good agreement with other reported data [9]. The observed secondary ion rates per incident primary beam particle and time resolution are currently not far from those of similar systems $[10,13]$. However, as mentioned above, absolute numbers for the detection efficiency of the ToF spectrometer cannot be deduced [21].

In studies of the electron start mode it was additionally seen that due to simultaneous extraction, detection efficiency and time resolution are not completely independent. A higher ion extraction field tends to improve time resolution, but lowers overall measurement efficiency because electron extraction worsens. Depending on the application, the system allows to choose a trade-off between measurement efficiency and spectroscopic resolution.

\subsection{Comparison to Pulsing mode}

For comparison of the novel electron start mode with conventional AMS beam pulsing the secondary ion mass spectrum of a solid PTFE sample measured in both modes is shown in Fig. 3. The measurements were conducted also with $28 \mathrm{MeV} \mathrm{Au}^{6+}$ primary ions. The difference in time and hence mass resolution is obvious. Even after careful optimization of the AMS pulsing system the time spread of the $\mathrm{H}^{+}$peak was 
never below 56 nanoseconds.

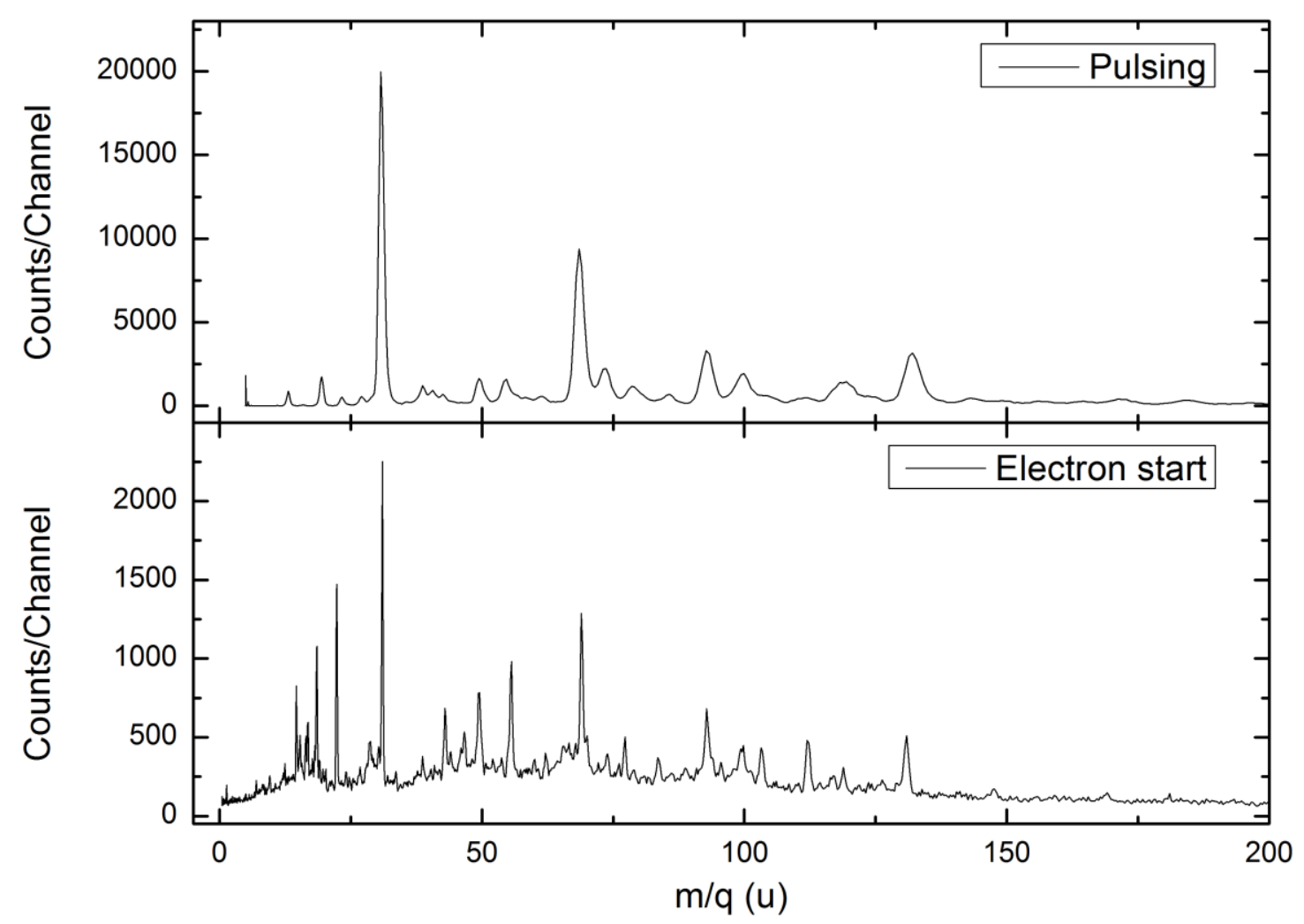

Fig. 3: Positive secondary ion mass spectrum of PTFE, compared for Pulsing and electron start measurement modes. Primary ion beam was $28 \mathrm{MeV}$ Au.

The signature peaks for positive ion SIMS spectra of PTFE [22] can be seen in both spectra; the expected combinations of $\mathrm{C}$ and $\mathrm{F}$ ion masses were identified and used for calibration of both spectra separately. Differences in relative peak heights arise from the differing resolution but also from the fact that the two spectra were not taken at the same time and from the same spot of the sample surface. In addition, counting statistics in the Pulsing spectrum are considerably higher due to a much longer measurement time. Since resolution in this mode is by far dominated by the beam pulse length, the time resolution is almost the same for the whole spectrum and mass resolution as a function of mass deteriorates proportional to the square root of the ion mass as expected from the time to mass conversion.

\subsection{Comparison to Transmission mode}

Furthermore, we evaluated the transmission detector pulses as a start signal for Time-of-flight measurements. The gas ionization detector only sees those primary particles which fully traverse the sample and do not scatter too far from the beam axis. This fraction strongly depends on the thickness and composition of the sample and can easily be determined by comparing count rates with the sample 
in and out of the beam path. However, the number of false starts is virtually zero in this mode as the signal to noise ratio of the gas ionization detector is extremely high.

In Fig. 4 a comparison of spectra taken with electron start and transmission mode is shown. The sample was a $2 \mu \mathrm{m}$ thick aluminum foil and measurements were conducted simultaneously on all three channels (electron and transmission detector as well as micro channel plate in the ToF spectrometer).

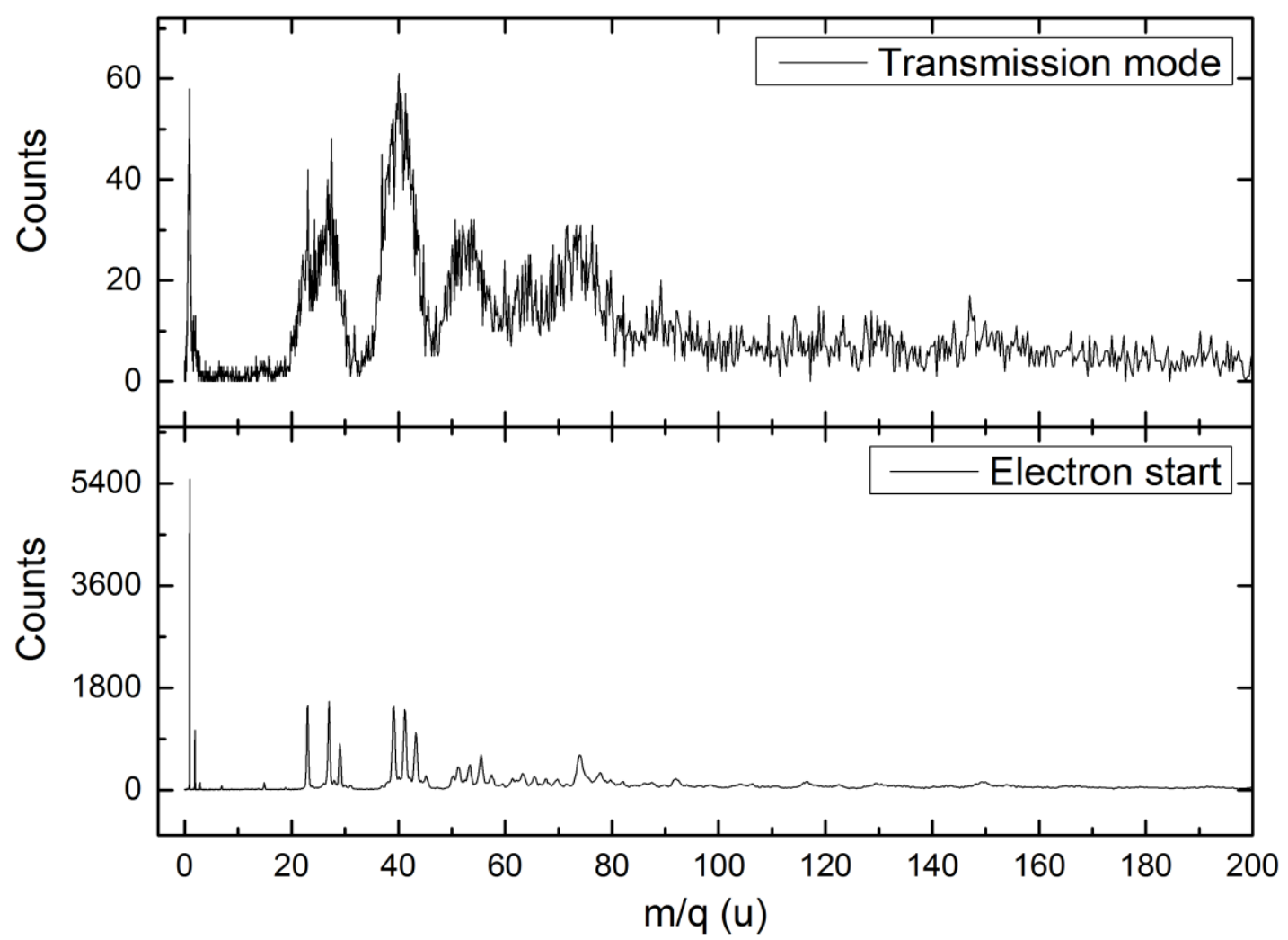

Fig. 4: Secondary ion mass spectra of an Al foil measured with 28 MeV Au primary ions in transmission and electron start mode simultaneously. The low count number in Transmission mode is due to high angular straggling in the 2 um thick foil.

The count rate in the transmission detector was much lower than in the electron detector due to significant angular straggling in the relatively thick foil. Mass resolution in the Transmission mode spectrum appears to be very poor and only groups of peaks can be identified. For the suspected $\mathrm{H}^{+}$peak a FWHM of 190 ns was determined in the time spectrum. Due to such bad resolution no reasonable selfcalibration of the spectrum was possible and the calibration from electron start mode, shifted by the delay between electron and transmission detector signals, was applied.

Slow charge collection in the gas detector and a long signal integration time in the associated electronics are the obvious reasons for the very large time jitter. The gas detector signal is detected more than $10 \mu \mathrm{s}$ after the signal in the electron detector with a fluctuation in the order of $200 \mathrm{~ns}$ (FWHM). Variations of the charge drift time in the detector gas and energy straggling of the transmitted primary ions can easily have a significant impact on time resolution. Under the current conditions the 
transmission detector is only useful for exact beam current monitoring and for Scanning Transmission Ion Microscopy (STIM) applications in imaging mode with thin samples.

\section{Conclusions and Outlook}

Here we presented first data taken with the new MeV-SIMS set-up at ETHZ, which proved functional in different ToF measurement modes. Best mass resolution was achieved by using a secondary electron start signal. This technique is novel in accelerator based MeV-SIMS with positive secondary ions.

In its prototype version the electron start mode reaches a mass resolution $\mathrm{m} / \Delta \mathrm{m}>100$ which is mainly limited by the initial energy spread of secondary ions. The effect of energy distribution on mass resolution is worsened by the low extraction field at the point of emission which prolongs the individual drift time of secondary ions into the acceleration field towards the ToF spectrometer. The situation can substantially be mitigated by the replacement of the simple linear ToF tube by a reflectron type mass spectrometer [23] which increases the total flight path and can provide at least a first order correction of the influence of the initial kinetic energy spread of ions. Additional optimization of the mass resolution and ion extraction efficiency can be expected from a higher extraction field strength which can be implemented by geometrical changes to the extraction region and by an increase of applied voltages. With all improvements a mass resolution $\mathrm{m} / \Delta \mathrm{m}>500$ should be within reach.

In the present state, the alternative Pulsing and transmission detector start modes cannot be used for recording mass spectra with reasonable resolution. However, they are useful for exact quantification of the number of incident beam particles, calculation of efficiencies and performance of STIM measurements. If needed, the performance of the Pulsing start mode can be improved by replacing the AMS pulsing system by fast vertical deflector plates [9]. To partly mitigate the effect of large beam losses when pulsing beams of rare particles such as cluster ions, the useful primary particle rate could be increased by combining a beam chopper with a bunching system [24].

The important added value of the secondary electron start mode is its very high duty cycle. A large fraction of incident beam particles produce a valid start signal and despite the unfavorable field configuration imposed by simultaneous extraction a large fraction of secondary ions is detected. For a typical sample, the rate of particles in the continuous primary beam is only about one order of magnitude higher than the net count rate in the mass spectrometer. This is a clear advantage over pulsed spectrometers if imaging under static SIMS conditions has to be performed. For pulsed beams or spectrometers, a large fraction of accelerated primary particles is lost. To obtain the same overall secondary ion count rate the beam current during the "on"-period of pulsing has to be higher inverse proportionally to the duty cycle. For MeV-SIMS this is at least 4 orders of magnitude. Therefore, the secondary electron start mode allows working with continuous beams of rare particles with extremely low intensity such as MeV molecular ions or clusters and in cases where beam intensity is lost by conventional submicron focusing or by collimation. This makes the electron start mode the best choice when using a low current primary cluster ion beam collimated by a capillary, as in our case.

This work was supported by the Swiss National Science Foundation under contract no. 156636 and Marie Curie Actions - Initial Training Networks (ITN) as an Integrating Activity Supporting Postgraduate Research with Internships in Industry and Training Excellence (SPRITE) under EC contract no. 317169. 


\section{References}

[1] R.W. Hutchinson, A.G. Cox, C.W. McLeod, P.S. Marshall, A. Harper, E.L. Dawson, and D.R. Howlett. Imaging and spatial distribution of beta-amyloid peptide and metal ions in Alzheimer's plaques by laser ablation-inductively coupled plasma-mass spectrometry. Anal. Biochem., 346(2):225-233, Nov 2005. [2] E.H. Seeley and R.M. Caprioli. Molecular imaging of proteins in tissues by mass spectrometry. Proc. Natl. Acad. Sci. USA, 105(47):18126-18131, 2008. DOI: 10.1073/pnas.0801374105.

[3] R.D. Macfarlane and D.F. Torgerson. Californium-252 Plasma Desorption Mass Spectroscopy. Science, 191(4230):920-925, 1976. DOI: 10.1126/science.1251202.

[4] P. Hakansson, A. Johansson, I. Kamensky, B. Sundqvist, J. Fohlman, and P. Peterson. Fast heavy-ion induced desorption of biomolecules. IEEE T. Nucl. Sci., 28(2):1776-1778, April 1981. DOI:

10.1109/TNS.1981.4331519.

[5] B.N. Jones, J. Matsuo, Y. Nakata, H. Yamada, J. Watts, S. Hinder, V. Palitsin, and R. Webb. Comparison of MeV monomer ion and keV cluster ToF-SIMS. Surf. Interface Anal., 43(1-2):249-252, Jan-Feb 2011. DOI: 10.1002/sia.3520.

[6] Y. Nakata, Y. Honda, S. Ninomiya, T. Seki, T. Aoki, and J. Matsuo. Matrix-free high-resolution imaging mass spectrometry with high-energy ion projectiles. J. Mass Spectrom., 44(1):128-136, Jan 2009. DOI: 10.1002/jms.1482.

[7] H. Yamada, Y. Nakata, S. Ninomiya, T. Seki, T. Aoki, J. Tamura, and J. Matsuo. MeV-energy probe SIMS imaging of major components in washed and fractured animal cells. Surf. Interface Anal., 43(12):363-366, 2011.

[8] M.J. Simon, M. Döbeli, A.M. Müller, and H.-A. Synal. In-air STIM with a capillary microprobe. Nucl. Instrum. Meth. B, 273:237-240, 2012. DOI: http://dx.doi.org/10.1016/j.nimb.2011.07.084.

[9] T. Tadic, I.B. Radovic, Z. Siketic, D.D. Cosic, N. Skukan, M. Jaksic, and J. Matsuo. Development of a ToF SIMS setup at the Zagreb heavy ion microbeam facility. Nucl. Instrum. Meth. B, 332:234-237, Aug 2014. DOI: 10.1016/j.nimb.2014.02.068.

[10] L. Jeromel, Z. Siketic, N.O. Potocnik, P. Vavpetic, Z. Rupnik, K. Bucar, and P. Pelicon. Development of mass spectrometry by high energy focused heavy ion beam: MeV SIMS with $8 \mathrm{MeV} \mathrm{Cl7+} \mathrm{beam.} \mathrm{Nucl.}$ Instrum. Meth. B, 332:22-27, Aug 2014. DOI: 10.1016/j.nimb.2014.02.022.

[11] F. Ames, M. Döbeli, C.R. Musil, P.W. Nebiker, L. Scandella, M. Suter, and H.A. Synal. Acceleration of clusters, collision induced charge exchange at $\mathrm{MeV}$ energies and applications for materials science. Nucl. Instrum. Meth. B, 112(1-4):64-67, 1996. DOI: http://dx.doi.org/10.1016/0168-583X(95)01136-6.

[12] R. Hill, P. Blenkinsopp, S. Thompson, J. Vickerman, J.S. Fletcher. A new time-of-flight SIMS instrument for 3D imaging and analysis. Surf. Interface Anal. 43: 506-509, (2011).

[13] Z. Siketic, I.B. Radovic, M. Jaksic, Hadzija M.P., and M. Hadzija. Submicron mass spectrometry imaging of single cells by combined use of mega electron volt time-of-flight secondary ion mass spectrometry and scanning transmission ion microscopy. Appl. Phys. Lett., 107(9):093702, 2015. DOI: http://dx.doi.org/10.1063/1.4930062.

[14] T.R. Ariyaratne, D.D.N.B. Daya, P. Hakansson, B.U.R. Sundqvist. Fast heavy ion induced photo emission from organic solids and plasman desorption mass spectrometry. Int. J. Mass Spectrom., 152:31-41, 1996. DOI: 10.1016/0168-1176(95)04327-6.

[15] M. Christl, C. Vockenhuber, P.W. Kubik, L. Wacker, J. Lachner, V. Alfimov, and H.-A. Synal. The ETH Zurich AMS facilities: Performance parameters and reference materials. Nucl. Instrum. Meth. B, 294:2938, 2013. DOI: http://dx.doi.org/10.1016/j.nimb.2012.03.004. 
[16] H.-A. Synal, G. Bonani, M. Döbeli, R.M. Ender, P. Gartenmann, P.W. Kubik, Ch. Schnabel, and M. Suter. Status report of the PSI/ETH AMS facility. Nucl. Instrum. Meth. B, 123(1-4):62-68, 1997. DOI: http://dx.doi.org/10.1016/S0168-583X(96)00608-8.

[17] H.A. Synal, G. Bonani, R.C. Finkel, Th.R. Niklaus, M.M. Suter, and W. Woelfli. The heavy ion injector at the Zurich AMS facility. Nucl. Instrum. Meth. B, 56-57, Part 2:864-867, 1991. DOI:

http://dx.doi.org/10.1016/0168-583X(91)95048-I.

[18] Scientific Instrument Services Inc. (SIS). Industry standard charged particle optics simulation software, (c) 2003-2006. URL: www.simion.com.

[19] A.M. Müller, M. Döbeli, M. Seiler, and H.-A. Synal. A simple Bragg detector design for AMS and IBA applications. Nucl. Instrum. Meth. B, 356:81-87, Aug 1 2015. DOI: 10.1016/j.nimb.2015.04.056.

[20] Technical Information Manual MOD. DT5751 2-4 CHANNEL 10 BIT 2-1GS/S DIGITIZER. CAEN, Revision N. 10 edition, February 2015. URL: www.caen.it/servlet/checkCaenManualFile?ld=10602.

[21] I.S Gilmore and M.P Seah. Ion detection efficiency in SIMS: Dependencies on energy, mass and composition for microchannel plates used in mass spectrometry. Int. J. Mass Spectrom., 202(1-3):217229, 2000. DOI: http://dx.doi.org/10.1016/S1387-3806(00)00245-1.

[22] G.L. Fisher, C. Szakal, C.J. Wetteland, and N. Winograd. ToF-SIMS evidence of intercalated molecular gases and diffusion-limited reaction kinetics in an alpha particle-irradiated PTFE matrix. J. Phys. Chem. B, 110(4):1820-1829, FEB 2 2006. DOI: 10.1021/jp055402d.

[23] B.A. Mamyrin, V.I. Karataev, D.V. Shmikk, and V.A. Zagulin. The mass-reflectron, a new nonmagnetic Time-of-Flight mass spectrometer with high resolution. Sov. Phys. JETP, 37:45-8, July 1973. [24] P.N. Ostroumov, V.N. Aseev, A. Barcikowski, B. Clifft, R. Pardo, S.I. Sharamentov, M. Sengupta. Beam test of a grid-less multi-harmonic buncher. Proceedings of PAC07, Albuquerque, New Mexico, USA 2007, C. Petit-Jean-Genaz (ed.), IEEE 2007, p. 2242 